\title{
The Aspects, the Origin, and the Merit of Aware Computing
}

\author{
Yasuji Sawada \\ Tohoku Institute of Technology, 35-1 Yagiyama-Kasumi, Taihaku, Sendai 982-8577, Japan \\ Correspondence should be addressed to Yasuji Sawada, sawada@tohtech.ac.jp
}

Received 24 February 2012; Accepted 2 May 2012

Academic Editor: Qiangfu Zhao

Copyright (C) 2012 Yasuji Sawada. This is an open access article distributed under the Creative Commons Attribution License, which permits unrestricted use, distribution, and reproduction in any medium, provided the original work is properly cited.

In this paper we tried to understand scientifically the awareness, a daily word. Some aspects of awareness, such as qualitative or quantitative, the targets of awareness, either the external world or the internal world, were discussed. Suggestion on the human awareness was described from the experimental results of visual hand tracking. The origin and the merit of awareness in the process of evolution of animals were discussed. Finally some characters of possible aware computers and aware robots were studied.

\section{Introduction}

For the scientists and the engineers to understand and to make use of the human ability, it is needed to translate into scientific terms, the words of human science expressing the human ability, which has been created in a long history. awareness is a word in human science. Now we are asking what it is explained in scientific terms.

Awareness in human science term naturally implies existence of a subject. There is no awareness in a system which has no subject. Subject implies existence of a central information processing system and an exterior self-expression device. There is no subject which has no mind and body. In the history of biological evolution, brain was originally created to produce information for body motion effective for survival against external change. A sensorial system was also created simultaneously to check the effectiveness of the motion.

In this paper, we survey how this word has been used in our daily life. We analyze them and try to extract essential factors and try to discuss them by scientific terms (Section 2). We also refer to the results of our recent experiments of visual tracking [1-4]. These experimental results suggested that two kinds of awareness exist in the human sensorial-motor system, which functions in the mutually exclusive manner (Section 3). Combination of the various aspects of the awareness, which we used in daily life and some experimental implication, has led us to believe that "usefulness" may be a keyword to understand the awareness and to apply the concept of awareness for the aware computers and aware robots. Thus, the paper is constructed in the following order:
(1) some aspects of awareness,

(2) experimental suggestions on the concept of awareness,

(3) usefulness of awareness,

(4) awareness of the internal world and mind,

(5) aware computer,

(6) an aware robot which behaves as if it had a free will.

\section{Some Aspects of Awareness}

In this section, we examine some sentences including awareness used in daily life and try to find some aspects among them.

\subsection{Qualitative and Quantitative. \\ "I am aware of following the preceding car within the distance of ten meters." (S1)}

This sentence means existence of a subject, an action, and an evaluation. The evaluation is quantitative. We may call this awareness "quantitative awareness." On the other hand, human civilization has sorted the complex phenomena of the external world into a countable number of the concepts. Awareness is also used to identify the kind of the body action with one of the known concepts. For example,

"I am aware of walking in spite of my original intention of running." (S2) 
We might call this kind of awareness as "qualitative awareness." These examples show that for the awareness of any kind, body motion seems indispensable as an exterior self-expression device of self. We examine if this is true in the sections below.

2.2. External and Internal. Being aware of external phenomena is daily, as

\section{"I am aware of snow falling outside the window."}

When we are aware of some phenomena which occurs with some distance from the observer's body, the target of awareness is external. However, it is important to realize the difference between (S3) and a sentence such as

\section{"A camera is ready to take the record of falling snow outside." (S4)}

The difference is essential for understanding an aspect of awareness, which we discuss in Sections 2.4 and 4 . Furthermore, it is also important to understand that the target of awareness is external even when the target of awareness is within our body.

\section{"I am aware of my stomach aching." (S5) \\ "I am aware of my skin hurting as the result of my falling down on the floor." (S6)}

One may think that the stomach is internal, whereas the skin is external. But there is no principal difference between the pain in the skin and the pain in the stomach, by assuming that all the body parts are external from the central information processor (CIP). As we mention later, even the memory system is external to the subject. In that sense, stomach is not internal. The stomach is painful because it was attacked by the external virus. Information of pain is sent to the CIP from a sensor system in a part of the body. This information was created to inform the damage of the parts of the body for survival. The information on the condition of the parts of the system is needed for the evaluation of the action. For survival purpose repair or replacement of the part may be important.

The targets of sensing a part of body (S5) and (S6) are considered also external.

\subsection{Awareness of Thinking.}

"I am aware of imagining the falling snow outside of the house." (S7)

Thinking or imagining is definitely internal. But the time when he imagines the falling snow and the time when he is aware of imagining it is not simultaneous. Most of the time, he may be imagining the falling snow, and this fact is constantly memorized in the memory system. From time to time, the CIP is switched from the imagination to the memory system, and he became aware he had been imagining up to this moment. The switching is done so smoothly and so quickly that he does not realize if the information is from past memory or real time. Although awareness is used in various ways, it can be unified by considering that the CIP is watching various parts of the external world including other body parts of the subject and even itself through the memory system.

2.4. Awareness and Experience. The awareness is identification of either observed phenomena or the image in the brain as one of the patterns experienced in the past. The awareness sometimes identifies information of the state of the local part of body, such as stomach, muscle, as one of the experienced patterns such as pain or itch. When we observe the dynamics of some object, we are aware what is going on. In contrast, a video-camera observes the same thing, but it is not aware what is going on. The difference clearly tells us that awareness is related to our knowledge.

When we say we are aware of something, the object of the awareness is either the phenomena in the external world, or the image of our internal world projected from the real world. In other words, we are never aware of the image which we never experienced. When the input signals into our brain are cutoff and when we are computing anticipatively what is going on in the external real world, the image is the imitation of the real world. Then, the brain commands the motor system to respond to the external world for adaptation or optimization.

2.5. Difference between the Awareness and Self-Monitoring. An important conceptual question arises: is the awareness different from self-monitoring? If a part failed and it was repaired automatically by a new part quickly enough to be in time for real-time processing, it is certainly a self-monitoring computer, but is it an aware computer?

Awareness is a kind of self-monitoring. But awareness is special in the sense that it monitors its own function by a CIP not by a distributed system. Any central monitor system observes the function of the total system by reducing information, either by identifying the pattern of function with one of the stored pattern, or by segmenting the total system into a relatively small number of groups and by identifying the type of mal-function with the stored pattern. The aware computer may say in the former case "I am aware that I am writing a paper." It may say in the latter case "I have a pain in my stomach.” No CIP can be aware that the 31586th cell in my stomach has trouble in calcium channel.

\section{Experimental Suggestions on the Concept of Awareness}

Hand tracking is the experiments [1-4] in which a subject is asked to follow by a cursor as accurately as possible a target moving on a screen programmed by a computer. When the target is shown it is a visual tracking experiment, while it is an intermittently blind target tracking when the target is intermittently hidden. Among various facts found in the tracking experiment, two facts are relevant here. In the visible tracking experiments, the cursor is always in 
an error-corrective mode, and therefore is retarded with respect to the target. On the other hand, the cursor moves in an anticipatory mode and precedes the target in the blind tracking [2-4].

A question we ask here is whether visible tracking mode and blind tracking mode are both the aware computing? This question was a start which has lead me to a general question this present paper is studying. It should be most natural to say that a visual tracking is a typical aware computing, because it is an experiment in which a subject's brain computes a proper hand motion which should minimize the error he is aware of with respect to an external target. On the other hand, it should be discussed whether a blind tracking experiment is aware computing or not. From the discussion in Section 2 we can say that this mode is also aware computing. The brain computes the hand motion to follow the hidden target using the memory of target motion observed in the visible region.

Another experiment proved that the same acceleration occurs even in a fully visible target tracking, if the target motion is fast [1]. This result was explained that the information processing speed of the vision system is not fast enough to take in the positional information incessantly, and that the percept-motor system is controlled to partly use the predictive mode instead of error-corrective mode. In addition, it is shown that two modes do not operate simultaneously. They are mutually exclusive. Why it has to be mutually exclusive? Is it possible to make a system which is aware of the both? This question is probably related to the question why internal world exists first of all. It might be that the internal world is created to compensate information which is not always available by some reason, for example, when the external motion is too fast that the visual system cannot process the information of the instantaneous information which evolves too quickly, as shown experimentally [1]. If a computing system is fast enough by using a fast processor, there may be no need to have an internal world.

When the speed of the external world is not very fast, one may think it may be possible to use both external information and the internal information at the same time. But it is not the case, because it must creates its own consistent body motion. For this purpose, CPS must have one and only one awareness at a time. They are thus mutually exclusive.

\section{Usefulness of Awareness}

What is the merit of aware computing? The awareness has been developed in biological system for survival against both external enemies and internal troubles. A number of perceptive systems and predictive capabilities were developed to protect biological systems from the external enemies, and a variety of feelings and internal neural systems were developed for warning of the possible internal system troubles.

Are they necessary for the artificial computer systems, too? We shall discuss this problem in a later section.

The sentences from (S1) to (S7) except (S4) used in daily life shown in the previous section include awareness, but does not always seem to be related to evaluation of his action.
Present human society is deviating from the severe biological evolutional society. Each action of individual human is not necessarily related to the survival evaluation and therefore, awareness under these circumstances is not accompanied by evaluation. It is not important for survival whether he runs or walks in an ordinary life. But it is certainly important if he is trying to escape from a tsunami. An aware system has advantage for surviving, because it can sometimes predict the external world and can give a chance to move to avoid it.

Awareness is the real-time knowledge on the evaluation of the action with respect to the effectiveness for survival.

\section{Awareness of the Internal World and Mind}

The awareness of the internal world discussed so far is closely related to the mind, which is also a deep human language, not a scientific one. How close we are now to the concept of mind? We discussed awareness of thinking and awareness of the status of the body parts, pain of the stomach, pleasantness of the whole body, and so forth.

Perhaps only remaining part of mind is the problem of free will.

Proactive hand motion was observed in tracking experiments [1-3]. When they found that their hand moved proactively with respect to an object which they were supposed to follow as accurately as possible, many subjects reported that they felt as if they were intending to lead the object. This results pushed me to imagine a possible physical interpretation of the "inversion of causality," which is against principles of physics, but necessary for understanding the "free will" of human being.

The mechanism of proactive motion was clarified by a recent intermittently visible tracking experiments [2]. When the target is visible and moves slow enough, hand motion is error corrective and retarded with respect to the target motion (error-corrective mode). On the other hand, when the target is moving invisibly on an already known orbit, the hand motion was found to precede the target. It was measured that the hand motion is accelerated as soon as the target is hidden. It was understood that the hand is then controlled by a predictive mechanism (predictive mode).

As discussed in a previous section, the awareness of the internal world may be created to compensate a slow processing of the human perceptive information. The preceding research [2] showed that the internal clock moved faster than the evolution of the real physical world, and the proactiveness of the body motion caused by the faster internal clock helps to optimize the dynamic error [1]. Internal world is the predicted projection of the external world using the information obtained in the past. This evolution of the internal world referring to the external world is a part of the activity in the brain called mind. The other part of the mind is the awareness of the local part of the body such as pain, discussed in Section 2.2.

\section{Aware Computer}

Already, most of the present computer systems are equipped with aware functions to some extent. One of the external 
enemies is virus, and antivirus vaccination software was developed greatly. Some alarm softwares informing of possible local trouble have also been developed. Nevertheless, the security of the computers equipped with these functions are monitored and taken care by human being not by the computer itself. Computers may be considered to be exposed to a severe survival society, unlike humans whose awareness is not directly related to survival. But we realize that a computer itself is not exposed to the severe market competition in real time. It is the manager of the computer company who is aware of the competition. In this sense, the computer is not a self-closed machine. It is not automotile either, like a future aware robot. When a computer is installed in a future robot which may move independently of the human control, the security must be controlled by a CPS of the robot. For this purpose, the concept of the self-closed real time aware computing will be indispensable.

From the following examples that we notice, it requires evaluation of the function and choice for some unknown factors for a computer or for a robot to be aware. The computer watches its own performance, and if the CPS of the computer itself can modify the system, either hardwares or softwares to improve the function, it is an aware computer. In other words, there can be no aware computer if there is no evaluation and action by the computer itself.

6.1. Self-Improving Computer. Let us imagine an "aware computer" which is designed to compute various optimization problems, and it is equipped with an evaluation counter whose number changes by the performance of the computer for constantly changing request. It is designed to change the system somewhat randomly, when the index of the evaluation counter goes down, and when the number of the counter becomes below a critical number, the power line is cutoff. Among an ensemble of computers which has a software and a hardware different from each other, a small number of computers with good performance will survive. In this case, if the computer can search and change the software by itself, constantly monitoring the number of the counter, it will be one of the aware computers.

6.2. Self-Sequencing Computer. Let us imagine a computer which performs a sequence of many programs by choosing out of many other sequences. If it has a memory which records the process and speed of computing, and if the computer from time to time stops computing and checks the previous process of computing, using the memory system, and if it can change the sequence of the job to achieve a higher global performance, then this computer might be called one of the aware computers.

\section{An Aware Robot Which Behaves as If It Had a Free Will}

Awareness implies existence of a CPS which can identify macroscopically the type of the present function with one stored. When an aware computer fails to identify the state of the machine, the computing system is not aware of what is going on.

It seems reasonable to define the aware computing as such having a CPS like ourselves. Some examples of sentiment we feel such as pleasantness, painfulness, happiness, and sadness are the awareness and identification of the present function of the system with one of the patterns we experienced in the past. When we say that the human being is an aware computer, how do we explain free will?

Even if we construct a very good aware robot which will monitor perfectly the macroscopic functions of the computer itself, it will not have free will. To implement free will, I propose here, based on the results of our experiments discussed in the Section 3, an aware robot with double time; one is the physical time and the other is a brain time. The robot is assumed to operate by the physical clock when the computer is functioning with external signal as a reference signal, and to operate with a brain clock which is a little faster than the physical clock when the computer is simulating the external world without the signals from the external world.

Such aware robot will find that his internal world is leading the real world when he compares both from time to time. He would feel that he is not following the change of the external world, but the world is following him. There are two conditions for this mechanism to work. One is that the dynamics of the world is simple enough that the computer can simulate it by learning. The second condition is that the brain clock moves faster than the physical clock when the external input is off. The experimental evidence [2-4] showed that this is really the case, when human is asked to track an object moving on a simple orbit.

\section{Epilogue}

Terms and concepts which the author discussed in the paper are

awareness,
external world,
internal world,
awareness of external world,
awareness of internal world,
mind,
free will,
self-closed system.

As the author mentioned in the Introduction, those words are terminologies in the human science domain, not in the natural science domain. The author tried in this paper to discuss them by the words of natural science, not to translate nor define them into natural science with the scientific precision, nor to create new terms which have strict definitions [5]. The terms in the human science have wide and various aspects because of the length of history it was used, compared to the terms used in the modern natural science. I believe it is more useful at present to discuss the 
aspects of the concept, not trying to define it in modern science terminology.

In the future, one will be able to make an aware robot which behaves as if it is implemented all the functions that a human has, and which stops functioning by cutting off the power when some number reaches to a critical value which is a measure of competition between the other robots. However, there remains a fundamental difference between the aware human and an aware robot. The former has a deep desire to keep living, while not for the latter.

Thus, we came finally to face a most fundamental question why we desire to live, and a question if we can implement the desire into an aware robot. At the present moment, we do not know why we desire to live. The only thing we know is these species which survived through a severe natural selection survived by obtaining the instinctive wish to live. It would be wonderful if we can understand this question either from genetic information or from brain structure. But, I am afraid that all these effort will fail.

Creators, by definition, whether it is a creator of a computer or the creator of animals design their products to survive as much as possible against their enemies. How have the creators of animals implemented the desire to live? I believe we can find some suggestions by translating this question into the scientific terms. To do so, we must look for the causal relation between the key word such as awareness, desire to live, natural selection, mind, and survival.

A following scenario seems most natural to the author.

(1) Animals have obtained awareness through the severe survival race through the evolution. Humans are most aware, hopefully.

(2) As a result, they obtained their internal world and mind.

(3) Then, they came to "think" that they have desire to live, because the mind they obtained was the kind which desire to live through the selection, although we do not know the physical mechanism of the desire yet.

(4) Important thing is to notice that it is not that we are aware because we have desire to live, but we desire to live because we are aware.

\section{References}

[1] F. Ishida and Y.E. Sawada, "Human hand moves proactively to the external stimulus; an evolutional strategy for minimizing transient error," Physical Review Letters, vol. 93, no. 16, Article ID 168105, 2004.

[2] Y. Hayashi, Y. Tamura, K. Sase, K. Sugawara, and Y. Sawada, "Intermittently-visual tracking experiments reveal the roles of error-correction and predictive mechanisms in the human visual-motor control system," Transactions of The Society of Instrument and Control Engineers, vol. 46, no. 7, pp. 391-400, 2010.

[3] Y. Hayashi, Y. Tamura, K. Sugawara, and Y. Sawada, "Why the hand motion proceeds the target in tracking experiment?" in Proceedings of the 3rd International Symposium on Mobiligence, vol. 34, 2009.
[4] Y. Hayashi and Y. Sawada, "A transition from an alternative error-correction mode to a synchronization mode in the mutual hand tracking and the mutual finger tapping," Physical Review E. Submitted.

[5] H. R. Maturana and F. J. Varela, Autopoiesis and Cognition, D. Reidel, Dordrecht, The Netherlands, 1980. 

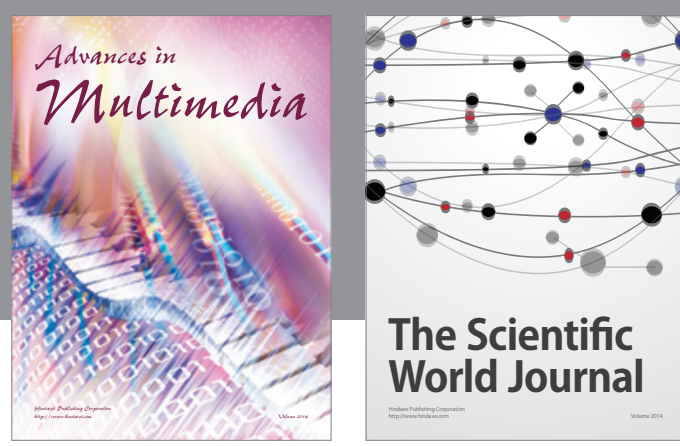

The Scientific World Journal
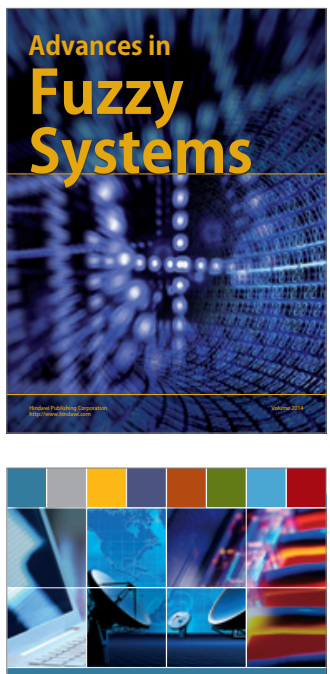

Computer Networks and Communications
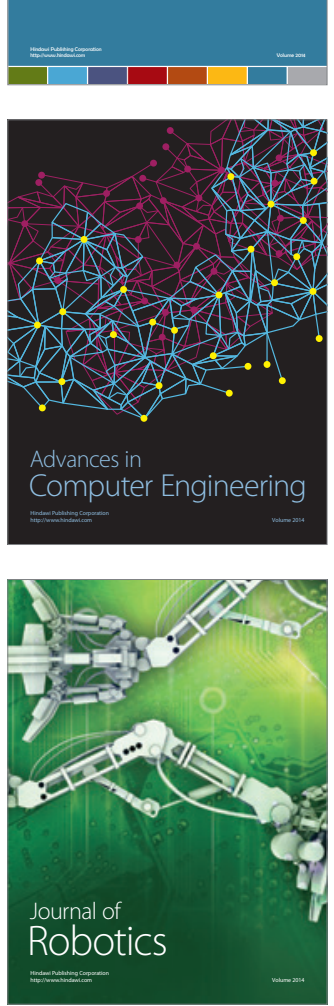
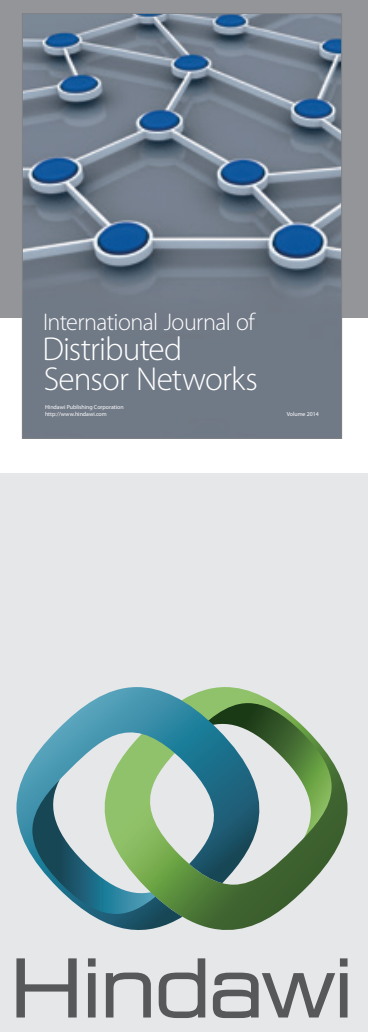

Submit your manuscripts at

http://www.hindawi.com
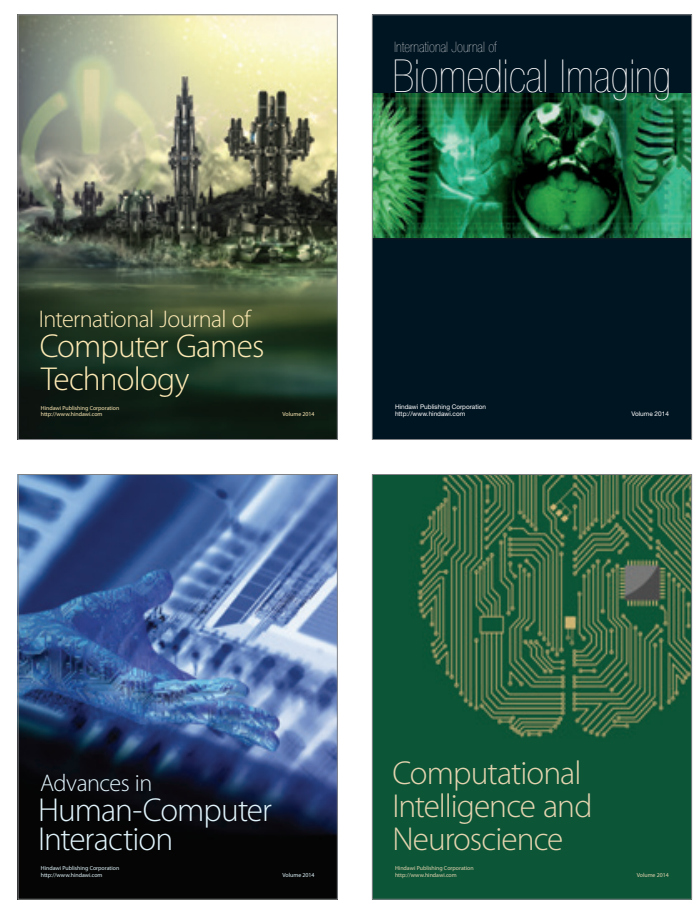
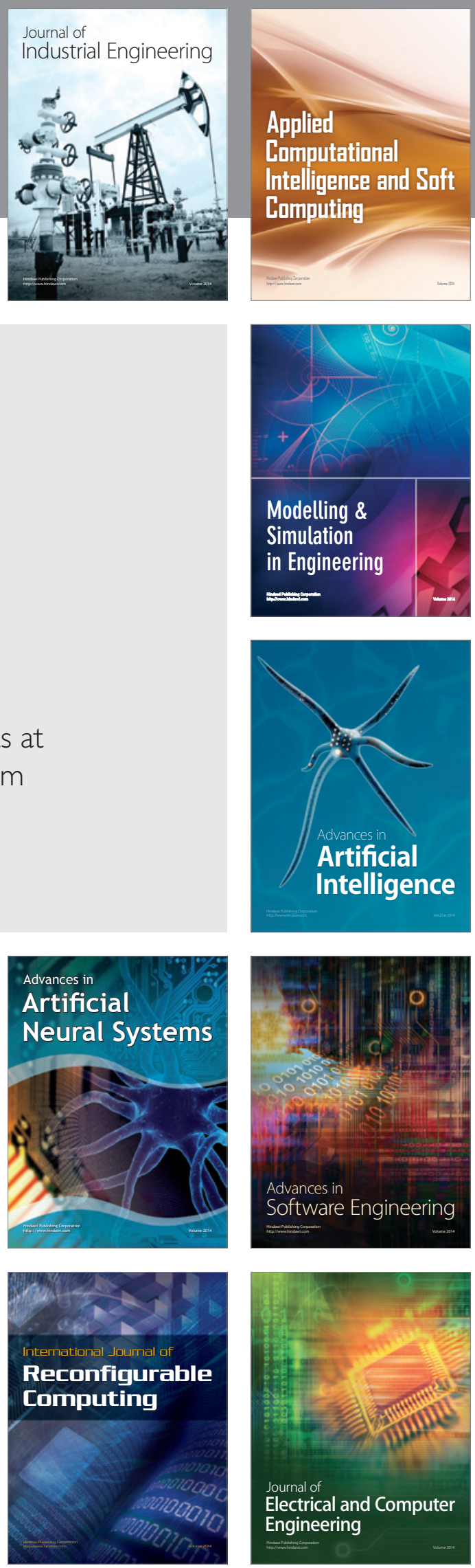